\title{
The effects of oviposition-site deprivation on Anopheles gambiae reproduction
}

\author{
Kathryne L Dieter, Diana L Huestis and Tovi Lehmann*
}

\begin{abstract}
Background: The African malaria mosquito, Anopheles gambiae, depends on availability of suitable surface water for oviposition. Short and long dry spells occur throughout the year in many parts of its range that limit its access to oviposition sites. Although not well understood, oviposition-site deprivation has been found to rapidly reduce egg batch size and hatch rate of several mosquito species. We conducted laboratory experiments to assess these effects of oviposition-site deprivation on An. gambiae and to evaluate the role of nutrition and sperm viability as mediators of these effects.

Methods: Anopheles gambiae adults ( $1-2 \mathrm{~d}$ old $)$ from the G3 laboratory colony were assigned to the following treatment groups: oviposition-deprived (fed once and then deprived of oviposition site for 7 or $14 \mathrm{~d}$ ), multiple-fed control (fed regularly once a week and allowed to lay eggs without delay), and age matched blood-deprived control (fed once, three days before water for oviposition was provided). Egg batch size and hatch rate were measured. In the second experiment two additional treatment groups were included: oviposition-deprived females that received either a second (supplemental) blood meal or virgin males (supplemental mating) 4 days prior to receiving water for oviposition.

Results: An. gambiae was highly sensitive to oviposition-site deprivation. Egg batch size dropped sharply to 0-3.5 egg/female within 14 days, due to reduced oviposition rate rather than a reduced number of eggs/batch. Egg hatch rate also fell dramatically to $0-2 \%$ within 7 days. The frequency of brown eggs that fail to tan was elevated. A supplemental blood meal, but not 'supplemental insemination,' recovered the oviposition rate of females subjected to oviposition-site deprivation. Similarly, a supplemental blood meal, but not 'supplemental insemination,' partly recovered hatch rate, but this increase was marginally significant $(P<0.069)$.

Conclusions: Even a short dry spell resulting in oviposition-site deprivation for several days may result in a dramatic decline of An. gambiae populations via reduced fecundity and fertility. However, females taking supplemental blood meals regain at least some reproductive success. If mosquitoes subjected to oviposition-site deprivation increase the frequency of blood feeding, malaria transmission may even increase during a short dry spell. The relevance of oviposition-site deprivation as a cue to alter the physiology of An. gambiae during the long dry season is not evident from these results because no reduction in hatch rate was evident in wild M-form An. gambiae collected in the dry season in the Sahel by previous studies.
\end{abstract}

Keywords: Anopheles gambiae, Dry season, Drought, Hatch rate, Malaria, Reproduction, Seasonality, Vectorial capacity, Oviposition, Fecundity

\footnotetext{
* Correspondence: TLehmann@niaid.nih.gov

Laboratory of Malaria and Vector Research, National Institute of Allergy and Infectious Diseases, National Institutes of Health, 12735 Twinbrook Parkway, Room 2W-09-C, Rockville, MD 20852, USA
} 


\section{Background}

Like most mosquitoes, Anopheles gambiae, the African malaria mosquito, depends on availability of suitable surface water for oviposition. However, during the dry season and dry spells during the wet season, larval sites may not be available for days, weeks, or even months in different environments [1-6]. Under such conditions, mosquitoes that develop eggs cannot lay them, a state hereafter referred to as "oviposition-site deprivation" [7], which differs from 'egg retention', a term applied also to an inseminated female which does not lay (some) developed eggs despite having access to a suitable oviposition site. The effect of oviposition-site deprivation on the physiology, behavior, and future life history of mosquitoes are scantly known, despite their possible ecological and public health implications. For example, the effects of oviposition-site deprivation on reproductive capacity, feeding rate, and longevity may correspondingly affect population size and disease transmission intensity during and following the period of deprivation. Moreover, not finding suitable larval sites may serve as a signal used by mosquitoes to switch from the typical reproductive state to reproductive depression in the dry season as previously described [8-14].

Only a few studies have evaluated the effects of oviposition-site deprivation on life-history traits. Reduced egg-batch size and hatch rate following oviposition-site deprivation was observed in Aedes aegypti, Ae. albopictus, Culex quinquefasciatus, An. maculatus, and An. pharoensis [7,15-19]. In these studies, the duration of oviposition-site deprivation varied from a mere 2 days (An. pharoensis) to 10 weeks (Ae. albopictus and $\mathrm{Cu}$. quinquefasciatus), however, statistical analysis was not always applied to the data. Both $A n$. pharoensis [15] and An. maculatus [18] revealed increased sensitivity of anophelines to short-term oviposition-site deprivation (2-5 d) compared with culicine species $(10-70 \mathrm{~d})$, as measured by their egg batch size and hatch rate over shorter periods of deprivation.

Here we evaluate the effects of oviposition-site deprivation on the egg batch size and hatch rate of $A n$. gambiae under laboratory conditions. We attempt to separate the effects of aging due to time and due to cumulative gonotrophic cycles from the oviposition-site deprivation itself and also evaluate the effect of sperm viability and the female nutritional state on the response to oviposition-site deprivation. Because this species experiences oviposition-site deprivation naturally, we hypothesized it would be less sensitive than other anopheline species previously examined. Our results suggest, however, that even short-term oviposition-site deprivation reduces egg batch size and hatch rate of $A n$. gambiae and that nutrition is a key mediator of this effect.

\section{Methods}

\section{Experimental design}

The G3 colony of An. gambiae was used throughout. Mosquitoes were reared under standard insectary conditions $\left(27^{\circ} \mathrm{C}, 75 \% \mathrm{RH}, \mathrm{L}: \mathrm{D} 12: 12 \mathrm{hrs}\right)$. Adults received fresh sugar solution daily. In each experiment, a total of 2,000 mosquitoes were divided, at 1-2 days old, into cages consisting of 100 females and 100 males to allow mating (Figure 1). Three cages were randomly assigned to the multiple-fed control, three cages for the oviposition-deprived group, and four cages for the blooddeprived group (two each for the once-fed on day 11 and once-fed on day 18 controls, Figure 1). When females were four days old (11 or 18 days for the once-fed controls), white leghorn chickens (Gallus gallus) were used to blood feed for twenty minutes; mosquitoes were provided with an additional twenty minutes if the feeding rate was $<90 \%$. Two-3-month old chickens were used to feed the mosquitoes in the cages, each one for a complete set of replicate cages (across all treatments). Unfed females were removed six hours after blood feeding.

On day seven, females to be assayed for oviposition (8-10 females/replicate cage of each treatment) were separated into individual $50 \mathrm{~mL}$ conical tubes lined with absorbent paper for egg laying and secured with netting on top to allow access to sugar pads. Males were also removed from all cages on day seven. Water for oviposition $(10 \mathrm{~mL}$ of dechlorinated tap water) was added individually to the tubes four days after blood feeding (no oviposition-site deprivation), 11 days after blood feeding (short-term: 7-day oviposition-site deprivation), or 18 days after blood feeding (long term: 14-day oviposition-site deprivation). Control groups included females that were blood-fed regularly (once a week on days 4, 11, and 18) and those that were blood-fed only once (four days before water for oviposition was provided). Egg dishes were placed in the cages of the multiple-fed controls to allow the remaining females to lay eggs. Females that did not receive a blood meal on day 4 were considered blooddeprived (BD). These groups were fed once on day 11 or 18 and were provided with water for oviposition four days after feeding. Every treatment was replicated two or three times in each experiment (Figure 1). A replicate is a set of all treatments in which females of a single cohort (same age) were housed in one cage/ treatment during blood feeding. Oviposition assays were performed on $8-10$ females per (replicate) cage in each time point (on day 8, 15, and 22, Figure 1), thus providing a sample size of $16-30$ per experiment. Hatch rate was measured for each egg batch that was laid.

Measures of egg batch size (EBS) and hatch rate were determined (see below). Females that died before their 


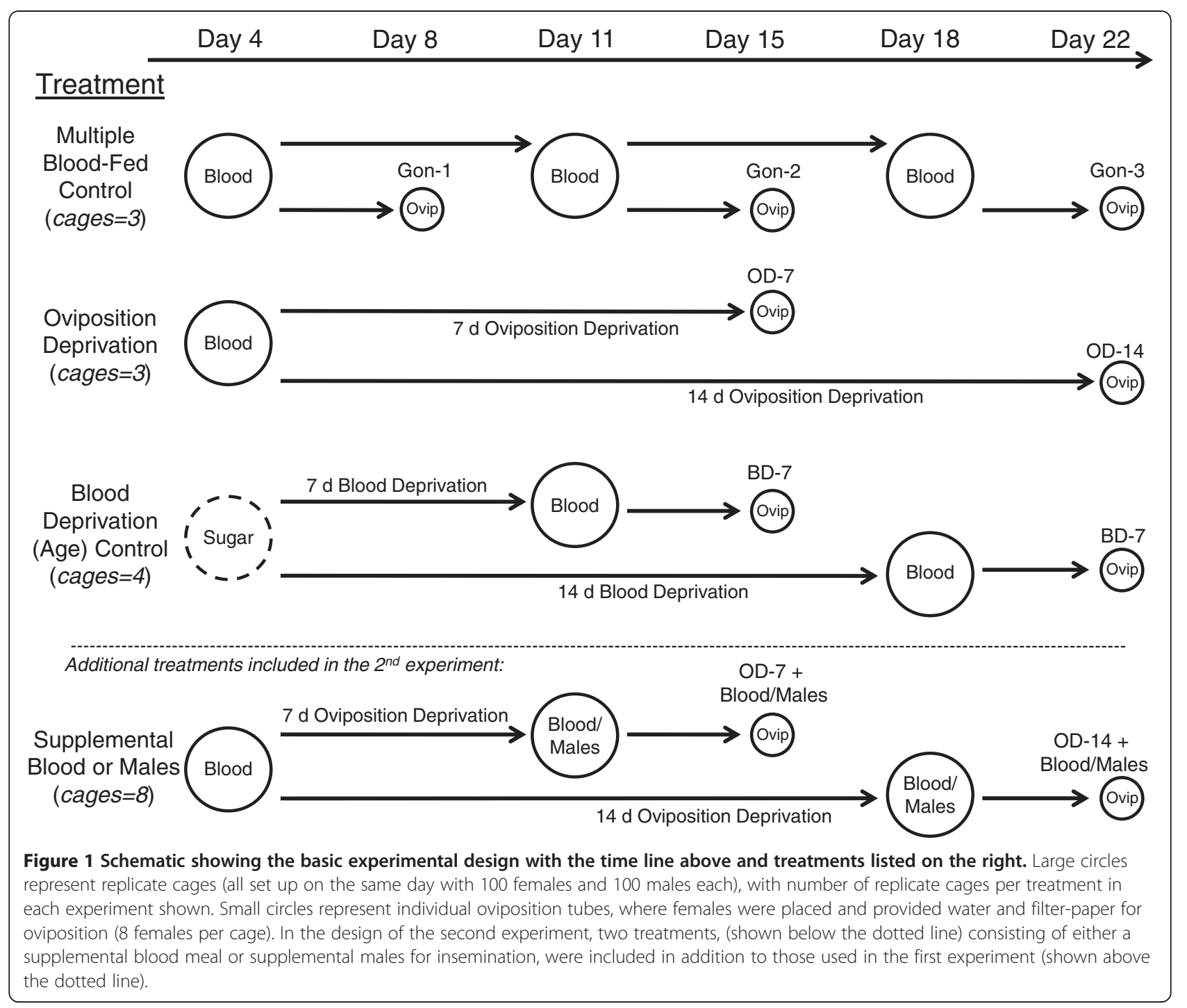

phenotype could be determined were excluded from analysis. The tubes were checked twice a day for three days; if eggs were laid, the female was killed and preserved and the appearance and number of eggs was recorded. Eggs that had sunk into the water were recorded separately and excluded from the count because they appeared abnormal and did not hatch in previous studies [20,21]. Eggs were then placed in labeled petri dishes and submerged in water. Hatch rate was recorded by transferring newly-hatched larvae onto filter paper via a glass pipette and counting them. Egg dishes were checked twice a day for three days to complete the estimate of hatch rate. Females that did not lay eggs or died during the experiment were dissected to determine their insemination status. Noninseminated females were excluded from the analysis. Mosquito body size was estimated using the measurement of wing length as previously described [9]. Only females with at least one intact wing were measured.

The second experiment was carried out as stated above, with the addition of two treatment groups (each replicated twice). One treatment consisted of oviposition-deprived females that were provided with a second (supplemental) blood meal 4 days prior to receiving water for oviposition. The other treatment consisted of oviposition-deprived females, which received 100 4-day-old virgin males as supplemental mating. The males were kept in the cage for 3 days until the females were separated into oviposition tubes. A random collection of males from the same cohort and cages was placed with virgin females to assess their mating vigor. Virtually all virgin females were inseminated after 3 days $(95 \%, \mathrm{n}=20)$. Individual females 
were separated and EBS and hatch rate were determined at the same time points as stated above.

\section{Ethics statement}

This study was carried out in accordance with the recommendations in the Guide for the Care and Use of Laboratory Animals of the National Institutes of Health. All animal procedures were approved by the National Institutes of Health Animal Care and Use Committee (ACUC, Protocol ID: LMVR102).

\section{Data analysis}

Statistical analyses were performed using SAS 9.2 (SAS Institute, Cary, NC). The effects of the treatments on EBS and hatch rate were evaluated using mixed model ANOVA (implemented using PROC MIXED) because both fixed-effect variables (e.g., oviposition-deprived groups vs. non-deprived control) and random variables (e.g., experiment and replicate nested within experiment) were included. If significant differences were indicated between treatments overall, contrasts were used to specifically evaluate the effect of oviposition-site deprivation compared with a subset of controls. Thus, to assess the effect of 7-day oviposition-site deprivation on EBS, contrasts were made between that group and the non oviposition-deprived controls which were in their $1^{\text {st }}$ and $2^{\text {nd }}$ gonotrophic cycle ( 1 contrast), and between the oviposition-deprivation group and the age-matched blood-deprivation group which received their first and only blood meal 3 days prior to the water for oviposition (1 contrast). The third contrast compared the EBS of the $1^{\text {st }}$ and $2^{\text {nd }}$ gonotrophic cycle of the non ovipositiondeprived controls. Because all females laid no (zero) eggs in some treatment/replicate combinations, resulting in non-estimable hatch rates, the variance component associated with 'replicate' could not be estimated in such cases. To determine differences in the percentage of females that laid eggs from different groups, log-linear categorical models were implemented using PROC CATMOD, which allows testing the same contrasts.

\section{Results and discussion}

The effect of oviposition-site deprivation on the reproductive output of An. gambiae was determined by comparison of EBS and egg-hatching rate of females subjected to short (7 days) and long (14 days) oviposition-site deprivation with corresponding values of non-deprived females of the same gonotrophic cycle as well as those of the same (chronological) age but in the next gonotrophic cycle. Additionally, ovipositiondeprived females were compared to females of the same chronological age and same gonotrophic cycle, which received their first blood meal 3 days before they were offered water for oviposition; the last group of females was deprived of a blood meal (blood deprivation). Unless otherwise stated, data obtained in the two experiments were analyzed together, treating experiments as random blocks (see Methods).

\section{EBS}

In both experiments, long-term oviposition-site deprivation (14 days) reduced mean EBS several fold, to near zero, compared with that of the first and second gonotrophic cycles (Exp. 1: 3.5 vs. 67 and 54 eggs/ female; Exp. 2: 0 vs. 45 and 37 eggs/female, respectively; Figure 2 and Table 1). However, mean EBS of 14-day oviposition-deprived females was not significantly smaller from that of 14-day blood-deprived females (12 and 1.4 eggs/female in experiments 1 and 2, respectively; Figure 2 and Table 1), suggesting that a nutritional deficit may mediate the effect of oviposition-site deprivation. Notably, mosquitoes in Exp. 2 were smaller (Additional file 1: Figure S1, $\mathrm{P}<0.001, \mathrm{~F}_{1,147}=150.8$ ), which probably explains their smaller overall EBS (across groups) and reduced the statistical power due to smaller differences between groups. Even short-term oviposition-site deprivation (7 day) reduced mean EBS approximately 2-fold compared with that of the first and second gonotrophic cycles (Exp. 1: 26 vs. 67 and 54 eggs/female; Exp. 2: 26 compared with 45 and 37 eggs/female, respectively; Figure 2 and Table 1). Mean EBS of 7-day oviposition-deprived females was significantly smaller than that of 7-day blood-deprived mosquitoes in the first experiment (61 eggs/female; Figure 2), whereas minimal difference was detected between these groups in the second experiment.

The proportion of oviposition-site deprived females that laid eggs (oviposition rate) was significantly reduced compared with non-deprived females, but the difference in mean EBS between the oviposition-site deprived and non-deprived females in those that laid eggs was minimal (Table 1 and Additional file 2: Figure S2). These results suggest that the overall effect of oviposition-site deprivation on EBS (including zero eggs) was mostly due to a lower oviposition rate rather than to a reduced number of eggs in those females that laid eggs. A high proportion of the oviposition-deprived females that did not lay eggs, especially those subjected to long oviposition deprivation, retained deformed brownish eggs that appeared to be undergoing resorption.

\section{Hatch rate}

In both experiments, nearly all females subjected to long (14 day) oviposition-site deprivation laid no eggs (see above), precluding estimating hatch rate for these groups. Thus, hatch rate was analyzed considering short-term (7-day) oviposition-site deprivation only. Remarkably, short-term oviposition-site deprivation 


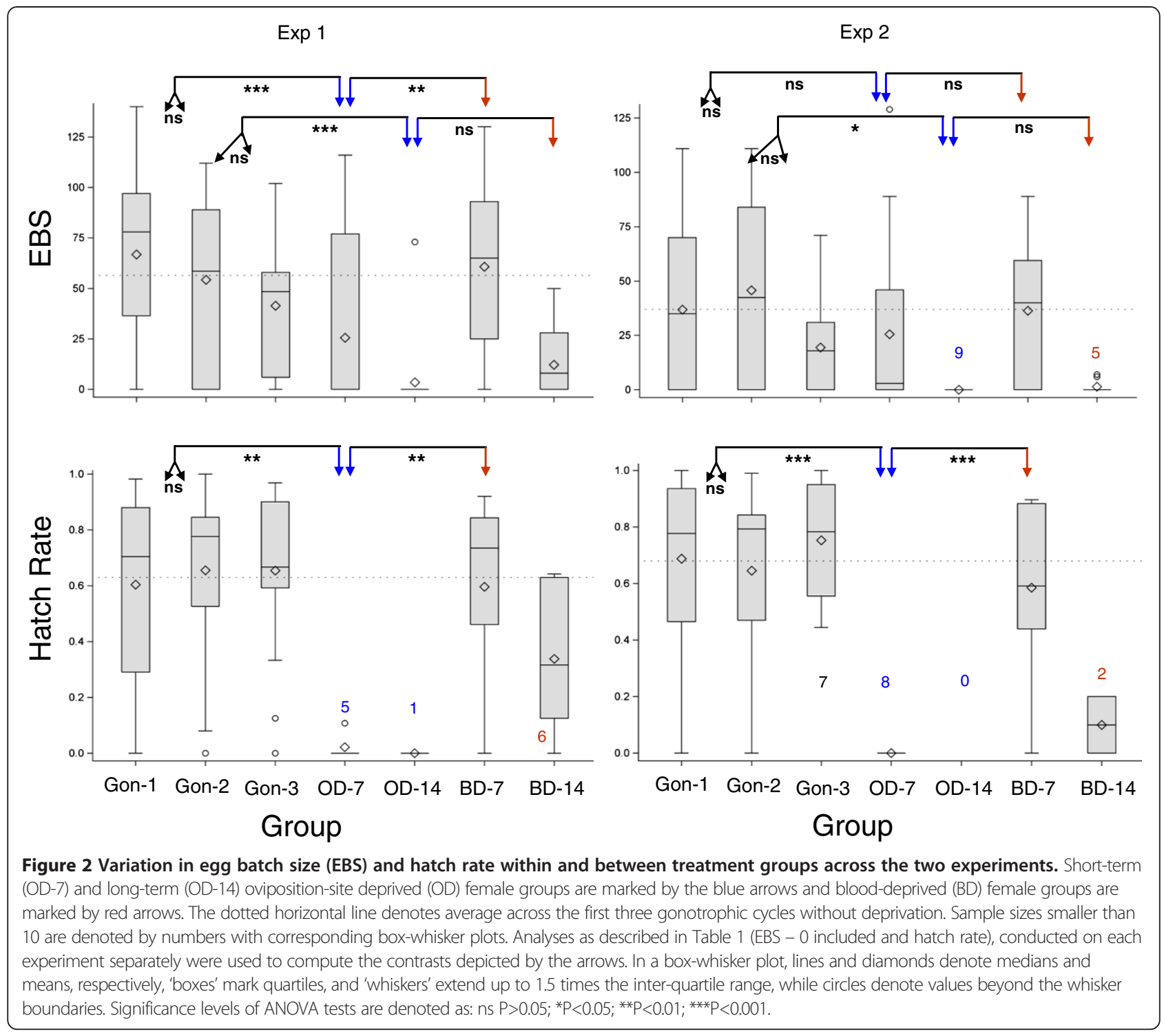

reduced hatch rate to $0-2 \%$ from $60-75 \%$ in both experiments (Figure 2 and Table 1). Associated with the reduced hatch rate was a high frequency of brownish eggs whose shell was sunk (Figure 3), similar to previous observations, e.g., [16]. However, many normal-looking eggs also failed to hatch.

\section{The roles of nutrition and sperm viability on egg batch size and hatch rate}

The underlying role of nutrition and sperm viability in mediating the effect of oviposition-site deprivation on EBS and hatch rate were evaluated in experiment 2 by providing oviposition-site deprived females with a supplemental blood meal or supplemental "insemination" via access to males, three days prior to provision of water for oviposition. The males used in the "supplemental insemination" treatment were randomly selected from others that mated successfully with virgin females. The oviposition rate of females subjected to long-term oviposition-site deprivation "recovered" to values typical of non-deprived females after they received one supplemental blood meal (the oviposition rate of supplemented females was slightly higher than that of non-deprived females), but not after they received "supplemental insemination" (Figure 4). The hatch rate after short term oviposition-site deprivation apparently increased from zero to $20 \%$, but this increase was only marginally significant $(\mathrm{P}<0.069$, Figure 4$)$. However, as with egg batch size, no increase in hatch rate was indicated following the supplemental "insemination" treatment (Figure 4).

\section{Conclusions}

The sensitivity of An. gambiae to short-term oviposition-site deprivation suggests that even short dry spells 
Table 1 The effect of short-term (7 days) and long-term (14 days) oviposition-site deprivation on egg batch size (EBS) and hatch rate

\begin{tabular}{|c|c|c|c|c|c|c|c|c|c|c|c|c|}
\hline & & EBS & & & EBS & 0 -exl & & & OvipRt $^{\text {b }}$ & & HR & \\
\hline Source & $D F$ & $Z / F$ & $P$ & $D F$ & $Z / F$ & $P$ & $D F$ & $x^{2}$ & $P$ & $D F$ & $F$ & $P$ \\
\hline Treatment group & $6 / 238$ & 12.1 & 0.001 & $6 / 138$ & 6.72 & 0.001 & 6 & 34.5 & 0.001 & $4 / 127$ & 12.9 & 0.001 \\
\hline Experiment & - & 0.56 & 0.29 & & 0.67 & 0.25 & 1 & 2.5 & 0.12 & $1 / 127$ & 0.31 & 0.53 \\
\hline Replicate(Experiment) & - & 0.77 & 0.22 & - & ND & ND & - & ND & ND & - & ND & ND \\
\hline Residual & - & 10.9 & 0.001 & & 8.31 & 0.001 & 6 & 6.8 & 0.34 & 127 & - & - \\
\hline 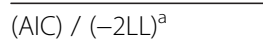 & - & (2433) & $(2437)$ & & 1354 & 1350 & & & & & & \\
\hline
\end{tabular}

Contrasts

\begin{tabular}{llllllllllll}
\hline OD-7 vs. Gon1 \& Gon2 & $1 / 238$ & 12.9 & $\mathbf{0 . 0 0 1}$ & $1 / 138$ & 0.01 & 0.91 & 11.9 & $\mathbf{0 . 0 0 1}$ & $1 / 127$ & 48.7 & $\mathbf{0 . 0 0 1}$ \\
\hline Gon1 \& Gon2 & $1 / 238$ & 0.23 & 0.63 & $1 / 138$ & 0.20 & 0.65 & 0.0 & 0.97 & $1 / 127$ & 0.03 & 0.86 \\
\hline OD-7 vs. BD-7 & $1 / 238$ & 7.0 & $\mathbf{0 . 0 0 9}$ & $1 / 138$ & 0.60 & 0.44 & 9.8 & $\mathbf{0 . 0 0 2}$ & $1 / 127$ & 31.7 & $\mathbf{0 . 0 0 1}$ \\
\hline OD-14 vs. Gon1 \& Gon3 & $1 / 238$ & 31.9 & $\mathbf{0 . 0 0 1}$ & $1 / 138$ & 0.04 & 0.83 & 16.8 & $\mathbf{0 . 0 0 1}$ & - & ND & ND \\
\hline Gon1 \& Gon3 & $1 / 238$ & 7.64 & $\mathbf{0 . 0 0 6}$ & $1 / 138$ & 15.9 & $\mathbf{0 . 0 0 1}$ & 0.0 & 0.95 & - & ND & ND \\
\hline OD-14 vs. BD-14 & $1 / 238$ & 0.6 & 0.44 & $1 / 138$ & 2.64 & 0.11 & 7.1 & $\mathbf{0 . 0 0 8}$ & - & ND & ND \\
\hline OD-7 vs. OD-14 & $1 / 238$ & 9.25 & $\mathbf{0 . 0 0 3}$ & $1 / 138$ & 0.04 & 0.84 & 7.0 & $\mathbf{0 . 0 0 8}$ & - & ND & ND \\
\hline
\end{tabular}

Mixed model ANOVA (Proc Mixed, SAS) was used to estimate variance among Experiments and Replicates (nested in Experiment) as well as Treatment group (fixed) on EBS. A log-linear categorical model (Proc Catmod, SAS) was used for oviposition rate. The effect of short-term oviposition-site deprivation on hatch rate was estimated using ANOVA (Proc GLM, SAS) using Experiment as random blocks. Hatch rate could not be estimated in females with long-term oviposition-site deprivation and blood deprivation because of very low oviposition rate (See also Figure 2 and the Methods for more details). Bold values were significant after accommodating the number of contrast tests (for each response variable separately) using the Sequential Bonferroni procedure [22].

${ }^{a}$ Model fit statistics residual -2 Log Likelihood and AIC values are given in parentheses in the DF column.

${ }^{b}$ Log-linear categorical models (Proc Catmod, SAS) were used to assess the effects of various factors.

(measured in days) during the wet season can reduce reproductive success, especially because of lower fertility (hatch rate) augmented by lower fecundity (egg batch size) due to a lower rate of oviposition. Similar sensitivity was reported for other culicids including An. pharoensis and An. maculatus [7,15-18]. High sensitivity to only a few days of oviposition-site deprivation is surprising for species that are often subjected to short (and long) dry spells under natural conditions, as is the case for An. gambiae. It is possible that a colony adapted to laboratory conditions has increased its sensitivity to oviposition-site deprivation because such deprivation is too rare in an insectary and it may also be related to other factors such as different blood source (chicken instead of human in our colony). However, the agreement across anopheline species (above), especially with the arid-adapted An. pharoensis, which was raised in the laboratory only three years prior to the study and was provided with human blood, suggests that the sensitivity to oviposition-site deprivation reflects the natural response of anophelines to such pressure.

However, oviposition-site deprivation was linked, as in most other studies listed above, with no (or limited) access to blood meals. The capacity to regain reproductive success by supplemental blood feeding suggests that, under natural conditions, mosquitoes may avoid the effects of oviposition-site deprivation by increasing their blood-feeding frequency. This hypothesis is consistent with the observations of gravid females that take additional blood meals $[14,23,24]$. Thus, malaria

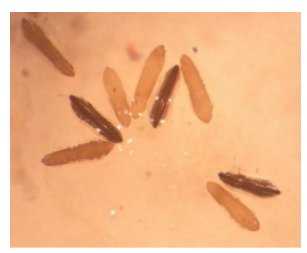

Normal tan eggs $(n=3)$ vs. brownish eggs after 7 d oviposition deprivation $(n=6)$

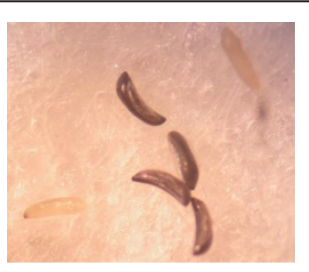

Normal tan eggs $(n=4)$ vs. white eggs after 14 d oviposition deprivation $(n=2)$

Figure 3 Eggs of females that experienced short (Left) and long (Right) oviposition-site deprivation with normal eggs of non-deprived females for comparison. Note that eggs fail to tan as the length of deprivation increases. Tests significance levels are denoted as in Figure 2. 


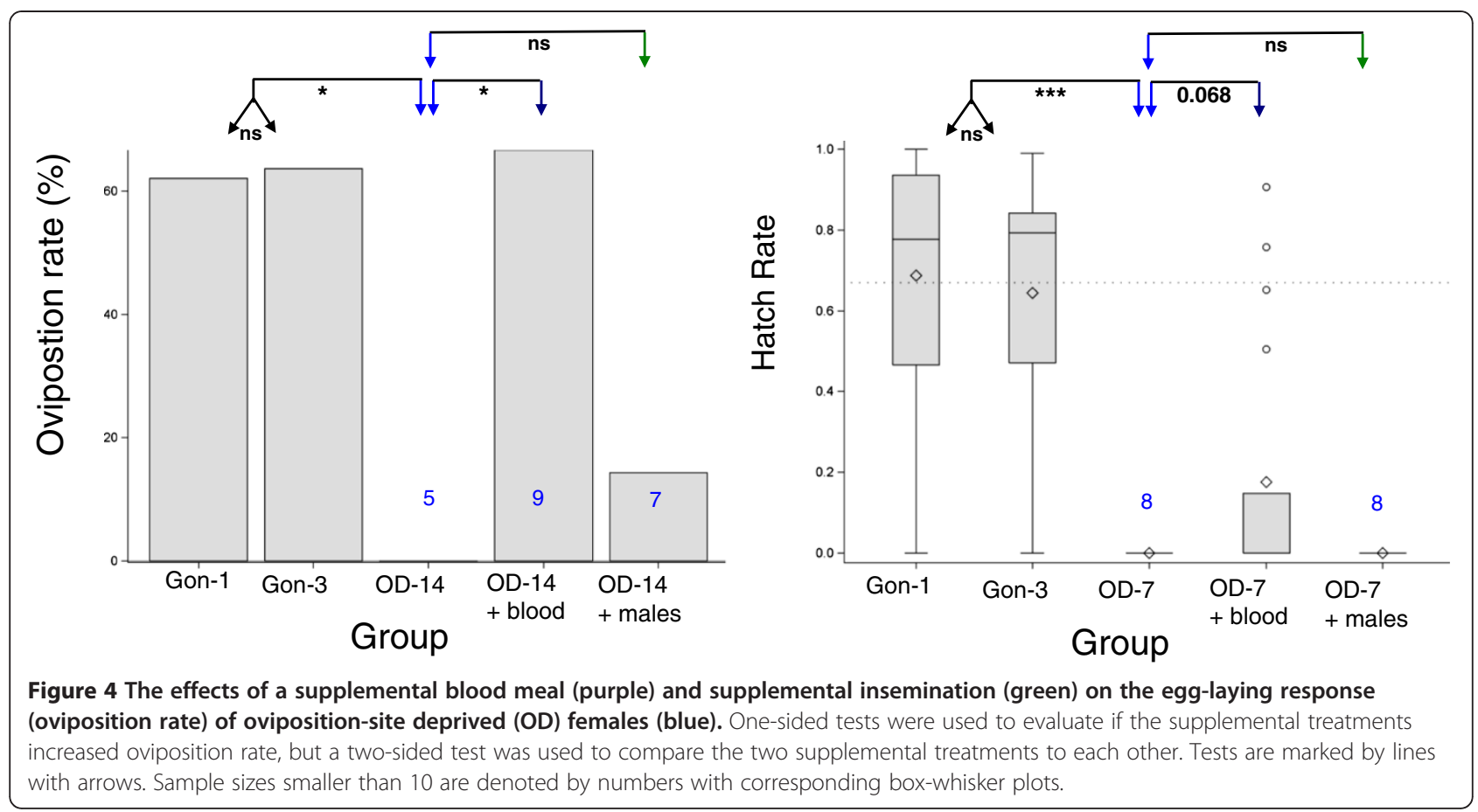

transmission may not decline (and might even increase) during a short dry spell. Additional studies measuring the blood-feeding rate of oviposition-site deprived $A n$. gambiae females are required to clarify that hypothesis.

The importance of oviposition-site deprivation as a cue to alter the physiology of $A n$. gambiae during the long dry season is not evident from these results. Similar to this study, Yaro et al. (2012) [14] reported a reduced oviposition rate in field-collected (blood-fed, semigravid, or gravid) females that were provided with water for oviposition once they reached the gravid state (without experimental deprivation) in dry-season females as compared with their wet-season counterparts. Presumably these dry-season females blood fed while they were naturally deprived of oviposition sites. Unlike the present study, the egg batch size for females that laid eggs was also lower in the Sahel [14] and a high frequency of brownish eggs was not observed [14]. Although their study did not empirically measure hatch rate, the dramatic reduction in hatch rate was not observed during the dry season (Yaro, Alpha and Dao, Adama - unpublished data). These observations suggest that during the long dry season in the Sahel, altered female physiology modifies the effect of oviposition-site deprivation on their reproductive output. This hypothesis requires additional study, as well as the effects of oviposition site deprivation on survival and on the vectorial competence (susceptibility) with human pathogens, as was recently studied in Culex pipiens quinquefasciatus and West Nile Virus [25].

\section{Additional files}

Additional file 1: Figure S1. Variation in body size, measured by wing length (WL), within and among treatment groups in the two experiments. The dotted horizontal line denotes the average WL across all groups of each experiment. Sample sizes smaller than 10 are denoted by numbers under corresponding box-whisker plots.

Additional file 2: Figure S2. Partitioning the effect of oviposition-site deprivation on overall egg batch size (EBS; upper panel), the number of eggs per batch for females that laid eggs (middle panel), and oviposition rate (lower panel). EBS of the treatment groups was averaged across experiments (see Figure 2 for results by experiment), following insignificant variance between experiments (see text and Table 1). Oviposition rate is shown separately for each experiment. Means of treatment groups with different letters are statistically different.

\section{Competing interests}

The authors declare that they have no competing interests.

\section{Authors' contributions}

KLD participated in the study design, carried out the experiments, and helped draft the manuscript. DLH participated in the study design and helped carry out various aspects of the experiments as well as draft the manuscript. TL conceived of the study, participated in its design and coordination, conducted the statistical analysis, and drafted the manuscript. All authors read and approved the final manuscript.

\section{Acknowledgements}

We thank Ms. Monica Artis for technical assistance during the laboratory work, Mr. Andre Laughinghouse and Mr. Kevin Lee for assisting with mosquito cultures, and Dr. Gwadz for facilitating the project. This study was supported by the Intramural Research Program of NIAID, NIH.

Received: 30 July 2012 Accepted: 23 September 2012

Published: 16 October 2012 


\section{References}

1. Simard F, Lehmann T, Lemasson JJ, Diatta M, Fontenille D: Persistence of Anopheles arabiensis during the severe dry season conditions in Senegal: an indirect approach using microsatellite loci. Insect Mol Biol 2000 9:467-479.

2. Beier JC, Copeland RS, Oyaro C, Masinya A, Odago WO, Odour S, Koech DK, Roberts CR: Anopheles gambiae complex egg stage survival in dry soil from larval development sites in western Kenya. J Am Mosa Control Assoc 1990, 6:105-109.

3. Charlwood JD, Vij R, Billingsley PF: Dry season refugia of malariatransmitting mosquitoes in a dry savannah zone of east Africa. AmJTrop Med Hyg 2000, 62:726-732.

4. Minakawa N, Githure Jl, Beier JC, Yan G: Anopheline mosquito survival strategies during the dry period in western Kenya. J Med Entomol 2001, 38:388-392.

5. Sogoba N, Doumbia S, Vounatsou P, Baber I, Keita M, Maiga M, Traore SF, Toure A, Dolo G, Smith T, Ribeiro JM: Monitoring of larval habitats and mosquito densities in the Sudan savanna of Mali: implications for malaria vector control. AmJTrop Med Hyg 2007, 77:82-88.

6. Toure YT, Petrarca V, Traore SF, Coulibaly A, Maiga HM, Sankare O, Sow M, Di Deco MA, Coluzzi M: Ecological genetic studies in the chromosomal form Mopti of Anopheles gambiae s.s. in Mali, West Africa. Genetica 1994, 94:213-223.

7. Yang P: Effect of oviposition site deprivation on oviposition performance and egg hatch rate of naturally blood-fed gravid Culex quinquefasciatus (Diptera: Culicidae). Proceeding of the Hawaiian Entomol Society 2008, 40:51-54

8. Adamou A, Dao A, Timbine S, Kassogue Y, Yaro AS, Diallo M, Traore SF, Huestis DL, Lehmann T: The contribution of aestivating mosquitoes to the persistence of Anopheles gambiae in the Sahel. Malaria J 2011, 10:151.

9. Huestis DL, Yaro AS, Traore Al, Adamou A, Kassogue Y, Diallo M, Timbine S, Dao A, Lehmann T: Variation in metabolic rate of Anopheles gambiae and A. arabiensis in a Sahelian village. J Exp Biol 2011, 214:2345-2353.

10. Huestis DL, Yaro AS, Traore Al, Dieter KL, Nwagbara Jl, Bowie AC, Adamou A, Kassogue $Y$, Diallo M, Timbine $S$, et al: Seasonal variation in metabolic rate, flight activity and body size of Anopheles gambiae in the Sahel. J Exp Biol 2012, 215:2013-2021

11. Lehmann T, Dao A, Yaro AS, Adamou A, Kassogue Y, Diallo M, Sekou T, Coscaron-Arias C: Aestivation of the African Malaria Mosquito, Anopheles gambiae in the Sahel. AmJTrop Med Hyg 2010, 83:601-606.

12. Omer SM, Cloudsley-Thompson JL: Survival of female Anopheles gambiae Giles through a 9-month dry season in Sudan. Bull World Health Organ 1970, 42:319-330

13. Omer SM, Cloudsley-Thomson JL: Dry season biology of Anopheles gambiae Giles in the Sudan. Nature 1968, 217:879-880.

14. Yaro AS, Traore Al, Huestis DL, Adamou A, Timbine S, Kassogue Y, Diallo M, Dao A, Traore SF, Lehmann T: Dry season reproductive depression of Anopheles gambiae in the Sahel. J Insect Physiol 2012, 58:1050-1059.

15. El-Akad AS, Humphreys JG: Factors Affecting oviposition and egg production in laboratory-reared Anopheles pharoensis Theobald. Bull SoC Vector Ecol 1988, 13:243-247.

16. Xue RD, Ali A, Barnard DR: Effects of forced egg-retention in Aedes albopictus on adult survival and reproduction following application of DEET as an oviposition deterrent. J Vector Ecol 2005, 30:45-48.

17. Wearne HM, Shisler JK: Stressed Versus Unstressed Oviposition Patterns In the Saltmarsh Mosquito, Aedes Sollicitans (Walker), (Diptera: Culicidae). Proc New Jersey Mosa Contr Assoc 1984, :115-119.

18. McDonald JL, Lu LC: Viability of mosquito eggs produced by female mosquitoes denied ovipositing sites. Mosa News 1972, 32:463-466.

19. Woke PA: Deferred oviposition in Aedes aegypti (Linn.). Ann Entomol Soc Am 1955, 48:39-46.

20. Yaro AS, Dao A, Adamou A, Crawford JE, Ribeiro JM, Gwadz R, Traore SF, Lehmann T: The distribution of hatching time in Anopheles gambiae. Malaria J 2006, 5:19.

21. Yaro AS, Dao A, Adamou A, Crawford JE, Traore SF, Toure AM, Gwadz R, Lehmann T: Reproductive output of female Anopheles gambiae (Diptera: Culicidae): comparison of molecular forms. J Med Entomol 2006 43:833-839.

22. Holm S: A simple sequentially rejective multiple test procedure. Scand J Stat 1979, 6:65-70.
23. Beier JC: Frequent blood-feeding and restrictive sugar-feeding behavior enhance the malaria vector potential of Anopheles gambiae s.l. and An. funestus (Diptera:Culicidae) in western Kenya. J Med Entomol 1996, 33:613-618.

24. Briegel $H$, Horler E: Multiple blood meals as a reproductive strategy in Anopheles (Diptera: Culicidae). J Med Entomol 1993, 30:975-985.

25. Smartt CT, Richards SL, Anderson SL, Vitek CJ: Effects of Forced Egg Retention on the Temporal Progression of West Nile Virus Infection in Culex pipiens quinquefasciatus (Diptera: Culicidae). Environ Entomol 2010, 39:190-194.

doi:10.1186/1756-3305-5-235

Cite this article as: Dieter et al:: The effects of oviposition-site deprivation on Anopheles gambiae reproduction. Parasites \& Vectors 2012 5:235.

\section{Submit your next manuscript to BioMed Central and take full advantage of:}

- Convenient online submission

- Thorough peer review

- No space constraints or color figure charges

- Immediate publication on acceptance

- Inclusion in PubMed, CAS, Scopus and Google Scholar

- Research which is freely available for redistribution 